\title{
Partial Discharges at Different Voltage Waveshapes: Comparison between two different acquisition systems
}

\author{
Pietro Romano ${ }^{1}$, Thomas Hammarström ${ }^{2}$, Tord Bengtsson ${ }^{3}$, Antonino Imburgia ${ }^{1}$, Antonino \\ Madonia ${ }^{1}$, Fabio Viola ${ }^{1}$ and Stanislaw M. Gubanski ${ }^{2}$ \\ ${ }^{1}$ L.E.PR.E. HV Laboratory, D.E.I.M. Department, Palermo University, 90128 Palermo, Italy, \\ ${ }^{2}$ Department of Materials and Manufacturing Technology Chalmers University of Technology, \\ SE 41296 Gothenburg, Sweden \\ ${ }^{3}$ ABB Corporate Research 72178 Västerås, Sweden
}

\begin{abstract}
In modern HV apparatuses the wide use of electronic converters, increase the stress on the involved insulation systems and thus affect the reliability of the whole power grid. Additionally, such non-sinusoidal voltage shapes contain high gradient flanks that create problems in the detection of partial discharge (PD) activity. The aim of this paper is to discuss the methodology on how to suitably approach PD detection in insulation systems exposed to various voltage waveshapes in general by comparing two different measuring systems. The first one, equipped with a resonant PD decoupler, designed specifically for detection at typical power electronic waveshapes and the other one, based on an antenna sensor with flat frequency response, a general-purpose $\mathrm{AC}$ system. It was found that not so much the acquisition principles as the features are important for effective detection of PD signals appearing at rapidly changing test voltages from power electronic devices.
\end{abstract}

Index Terms — Partial discharges, measurements, Square voltage waveforms.

\section{INTRODUCTION}

Increased use of power electronic devices based on Insulated-Gate Bipolar Transistors (IGBTs), Thyristors (SCRs) and Mosfets (FETs) results in the appearance in power systems of voltage waveforms that are different from sinusoidal and often contain fast transients. Exposure of electrical devices to such voltage waveforms yields new types of problems, principally in their insulation systems, and much work has been dedicated to evaluating these effects [1-7]. In the case of standard $50-60 \mathrm{~Hz}$ sinusoidal sources, the partial discharge (PD) detection techniques have taken advantage of the substantial difference in the frequency content between the driving voltage source and the discharge signals. This possibility disappears in the presence of fast transients, which have frequency content similar to that of PDs [8].

In this context new approaches and detection methods are required [9]. It is well recognized that Phase Resolved Partial Discharge (PRPD) patterns obtained under sinusoidal excitation can be useful for defect identification [10]. In the case of power devices supplied by synthesized sine waves, PDs are often concentrated to the voltage flanks and thus the pattern follows the Pulse Width Modulation (PWM) shape and the defect identification requires other

Manuscript received on 28 April 2017, in final form 24 December 2017, accepted xx Month yyy. Corresponding author: P. Romano. interpretation schemes. A quantitative interpretation scheme of the PWM waveform filter level (smoothness), yielding the same level of PD activity as for sinusoidal waveforms, has been defined [9]. As repetitive short rise-time pulses generated by most power electronic components cause an additional stress [11-15], the combined impact of these stresses makes the measuring process of PDs more difficult, especially in online conditions where additionally the external noise affects the measurements. Reduction of insulation lifetime is often associated with the presence of short rise-times in the supply voltage [9, 16-19]. Many papers are focused on the effect of rice time considering that some technical standards do not require the use of PWM waveforms (but only sinusoidal) for tests on components powered by inverters $[15,16,20,21]$.

This paper discusses some of the challenges involved in electrical PD detection when square voltage shapes are used. The ambition is to illuminate the requirements on a PD detection system that can be used in such cases, and illustrate it by comparing simultaneous measurement results of two different measurement systems, of which one is designed specifically for the detection at voltage waveforms generated by power electronic devices whereas the other is a general-purpose antenna based system designed for convenient field use. The waveforms generated by power electronic devices normally have a rise time of some microseconds, for GTOs, some tens of nanoseconds for 
IGBTs, passing through the BJTs that have intermediate rice times $[22,23]$. In addition, the actual sources generate pulse trains (PWM) of varying amplitude and/or duration to synthesize an AC waveform. If such waveforms had been used, the general-purpose system would show severe difficulties in performing the measurements and thus no detailed observations could be obtained. Instead, waveshapes that are close to the limit of the generalpurpose system capability (few microseconds) were used for illuminating the encountered difficulties as well as for demonstrating the limit of detectability in the laboratory and onsite.

\section{HIGH VOLTAGE GENERATION SYSTEMS}

The first setup is based on a high voltage switch (Behlke HTS 501-03-GSM), with the main objective to generate square shaped voltages of different rise times. Here the generic test object (illustrated as a cavity) is connected to point 'A' of the supply system, as indicated in Figure 1.

The second setup, a traditional AC IEC 60270 PD test circuit, employs a $50 / 60 \mathrm{~Hz} \mathrm{HV}$ transformer, a filter resistor of $100 \mathrm{k} \Omega$ to limit power system noise and a coupling capacitor of $100 \mathrm{pF}$ connected in parallel with the PD source. More details about both these setups can be found in $[7-9]$.

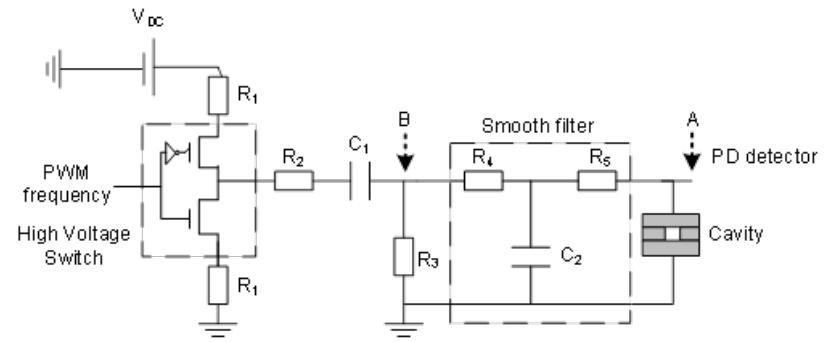

Figure 1. PD test setup with high voltage switch (Behlke HTS 501-03GSM) and the smoothing filter.

\section{ACQUISITION SYSTEMS}

Applying the IEC 60270 based PD detection method does not work at steep voltages, as the voltage remnant from the polarity shift overloads the PD detector [8] because the difference in frequency content is much lower than when using $50 \mathrm{~Hz}$ sinusoidal excitation. This method is therefore not used by the two acquisition systems in this work. The first system uses a resonant PD decoupler designed specifically for measurement of PDs at square shaped voltage waveforms, including PWM waveforms, and employs a stochastic PD detection technique [2]. The other system is based on an antenna sensor with flat frequency response and is mainly designed for online PD measurements. Additionally, the principles how the data are acquired and processed are based on different approaches, each one characterized by own pros and cons, as will be explained in the following. Finally, in the performed measurements, no common calibration process between charge and amplitude of the acquired signals is carried out, instead the focus was on suppressing the influence of noise and to perform relative measurements between the two systems.

\subsection{THE STOCHASTIC PD DETECTION SYSTEM}

The STOchastic PD detection system, (STOPD) is described in depth in $[8,9]$. The data processing technique utilizes the stochastical nature of the PDs to separate them from the high voltage remnants remaining from the applied waveform as well as phase locked disturbances. This requires full period acquisitions, which also allows a wide range of noise reduction techniques, not only filters. In particular time based noise suppression can be used.

The analog detection system, based on a Resonant PD Decoupler, consists of three different parts: a high pass filter, a frequency dependent voltage divider and finally the amplification part that is realized by utilizing the resonance peaks appearing in a coaxial cable with optimally mismatched termination impedances. This implies an approximate cut-off frequency of $10 \mathrm{MHz}$ [9]. To collect the data used in this paper, the decoupler is connected to a 14 bit NI-DAQ digitizer with a sampling rate of $100 \mathrm{MS} / \mathrm{s}$. A larger bit depth of the digitizer increases the dynamic range by detecting both large and small PDs, which is important for studies of insulation deterioration effects. The data processing principles utilized and described in [9] also allow the use of antenna sensors.

For the stochastic PD system, there is no hardware PD trigger, as generally an entire period is recorded and the PD trigger level is found by estimating the noise. Thus this system can set the trigger level automatically. The estimated sensitivity of the STOPD system is approximately $1.5 \mathrm{mV} / \mathrm{pC}$. For any of the configurations based on capacitive coupling, the shape of the PD response depends on the electrical filters utilized. An approach to avoid this issue is discussed in [9].

\subsection{SYSTEM BASED ON ANTENNA PD SENSOR}

For PD measurements, especially in online conditions, the use of inductive or capacitive sensors requires direct electrical connection to the devices under test. It usually implies a shutdown of the plant and non-negligible additional work. The use of an antenna sensor, designed to pick-up the electromagnetic field radiated by PDs, overcomes these problems and simplifies the measurements. Various antenna sensors have been proposed in literature [24-25], but often a reduced sensitivity in terms of directionality and bandwidth compared to traditional sensors, and a larger influence of surrounding noise limits their usefulness. Furthermore, antenna sensors do not provide information on the $\mathrm{AC}$ voltage phase, which has still to be obtained in another way.

The PD measurement system adopted in this work consists of a Portable Antenna Sensor (PASPD), also capable to sense the AC voltage field, integrated with a digital acquisition system. This system is connected via an integrated $\mathrm{Wi}-\mathrm{Fi}$ router to a computer, showing the acquired signals in real-time.

To overcome sensitivity problems and obtain noise rejection, sensors conventionally employ resonance 
antennas. In this particular case, however, an ultra-wide band (UWB) antenna is adopted, having an almost flat response in the VHF bandwidth of $0.1-100 \mathrm{MHz}$ where most of the PD radiated energy is generally observed. This novel approach allows obtaining a less disturbed pulse waveform, unlike the resonant sensors, thus simplifying the separation of pulses of different origin from noise by postprocessing techniques. All pulse signals are acquired, stored, processed and sent to the computer by a Field Programmable Gate Array (FPGA). Samples are acquired with a resolution of 8 bit and a rate of $200 \mathrm{MS} / \mathrm{s}$. The resulting bandwidth of the instrument (sensor + analog front-end + ADC) is $0.7 \mathrm{Mhz}-100 \mathrm{Mhz}[26,27]$.

The system acquires the pulses with the corresponding phase angle and records the time sequence. It does not return information about position within the total acquisition time. In particular, a spherical monopole placed at a short distance from a rear ground plane was adopted to improve the directional sensitivity of the PASPD system and its behavior is described extensively in [28-32]. Furthermore, no calibration process of the acquired signals is carried out, even though, from previous experiences, the estimated sensitivity of the PASPD system is approximately $10 \mathrm{mV} / \mathrm{pC}$ [33].

\section{MEASUREMENTS}

The choice of test objects has been made primarily to evaluate the effect of different input voltage waveforms on the PD characteristics and whether the chosen methods would detect these influences. Test objects that will produce either large or small as well as a few or many PDs have been selected to really be a challenge for the tested methods. A secondary aspect is to investigate the possibility to recognize a specific PD source (internal, surface) also when the PD pattern is generated by square waves of different rise times.

With this in mind, the PD sources were chosen to cover typical defects that normally appear in high voltage insulation systems, such as internal cavity (represented here by a three layer structure with a hole of $4 \mathrm{~mm}$ of diameter in the central layer) and surface discharge object (twisted pair). Finally, a three phase motor stator is chosen for representing a practical and more geometrically complex insulation system. The latter provides a multitude of PD sources and poses different difficulties for the evaluated detection methods.

Several test voltage shapes were used, a pure sinusoidal $50 \mathrm{~Hz}$ input was chosen as a reference for interpretation of the recorded PD activity. Four variants of square wave voltages were used with frequencies of 50 and $350 \mathrm{~Hz}$, each with a relatively fast rise time of $10 \mu \mathrm{s}$ or a slow rise time of $250 \mu \mathrm{s}$. Measurement data for each of the cases were collected with both acquisition systems, maintaining the same conditions during the measurements. For the STOPD system, complete traces, each covering one period, were recorded continuously [9]. The PASPD system was placed $40 \mathrm{~cm}$ away from the source with the antenna sensor oriented identically in all the cases. The signals were acquired by considering a statistically suitable number of pulses to record, not a specific number of cycles or time interval. The discharge amplitudes are therefore, for relative comparisons, indicated in $\mathrm{mV}$. Phase Resolved Partial Discharge (PRPD) patterns were acquired for each source of discharges.

\section{DATA ANALYSES AND INTERPRETATION}

\subsection{SINUSOIDAL VOLTAGE WAVEFORMS}

The conventional interpretation of the tested PD sources was made by applying a sinusoidal voltage of $50 \mathrm{~Hz}$. Tables 1 and 2 present respectively the parameters obtained by means of STOPD and PASPD systems. In Table 1, the input voltage amplitude, selected to be $10 \%$ larger than PDIV for each defect, the maximum and minimum value of PD amplitude detected by STOPD system are reported for each case separately, where Summed PD means the sum of all collected PD magnitudes per cycle (which is $20 \mathrm{~ms}$ in sinusoidal $50 \mathrm{~Hz}$ test), Average max $P D$ means the average of the maximum PDs found in each trace during the data collection. Finally, $\min / \max P D$ shows the $\max$ and $\min$ amplitudes found during the measurements. The STOPD system is able to determine the same parameters also for the square voltage shapes, unlike the PASPD system that is able to obtain them only for sinusoidal waveforms.

TABLE 1. PD data acquired at sinusoidal voltage of $50 \mathrm{~Hz}$ by STOPD

\begin{tabular}{c|c|c|c|c}
\hline \multicolumn{2}{c|}{ Input } & \multicolumn{3}{c}{ PD Sources } \\
\hline \multicolumn{2}{c}{ Sinusoidal 50 Hz } & Internal & $\begin{array}{c}\text { Twisted } \\
\text { Pair }\end{array}$ & Motor Stator \\
\hline $\begin{array}{c}\text { Voltage } \\
\text { Amplitude }\end{array}$ & {$[k V p p]$} & 14 & 2.2 & 4.8 \\
\hline $\begin{array}{c}\text { Min/Max } \\
\text { PD } \\
\text { amplitude }\end{array}$ & {$[m V]$} & $1.16 / 150$ & $18 / 280$ & $0.74 / 27$ \\
\hline $\begin{array}{c}\text { Average } \\
\text { Max PD }\end{array}$ & {$[m V]$} & 65 & 120 & 17 \\
\hline $\begin{array}{c}\text { Summed } \\
\text { PD/cycle }\end{array}$ & {$[\mathrm{V}]$} & 0.22 & 0.74 & 0.4 \\
\hline Number of & {$[P D s / \mathrm{cycle}]$} & 6.7 & 14 & 136 \\
\hline
\end{tabular}

In Table 2, PD parameters acquired with PASPD are shown. In addition to the discharge parameters, the instrument determines statistical parameters of the Weibull distribution calculated online for positive and negative discharges.

Table 2. PD data acquired at sinusoidal voltage of $50 \mathrm{~Hz}$ by PASPD

\begin{tabular}{c|c|c|c|c}
\hline \multicolumn{2}{c}{ Input } & \multicolumn{3}{c}{ PD Sources } \\
\hline \multicolumn{2}{c}{ Sinusoidal 50 Hz } & Internal & $\begin{array}{c}\text { Twisted } \\
\text { Pair }\end{array}$ & Motor Stator \\
\hline $\begin{array}{c}\text { Voltage } \\
\text { Amplitude }\end{array}$ & {$[\mathrm{kVpp}]$} & 14 & 2.2 & 4.8 \\
\hline $\begin{array}{c}\text { Min*/Max } \\
\text { PD } \\
\text { amplitude }\end{array}$ & {$[\mathrm{mV}]$} & $60 / 150$ & $40 / 270$ & $10 / 80$ \\
\hline Number of & {$[P D s / c y c l e]$} & 5.6 & 9.8 & 80.6 \\
\hline
\end{tabular}

* Minimum values are influenced by the trigger level used to prevent the acquisition of small-amplitude noises.

Starting from the assumption that a direct comparison between the values obtained from the two instruments can be difficult due to the completely different acquisition 
techniques, one can observe from the tables that the determined PD parameters show differences in the amount of PDs per cycle and their minimum amplitudes, which is partially caused by the selected trigger level. The maximum PD amplitudes are on the other hand quite similar for the two systems.

\section{Cavity PD acquisitions}

The acquired PRPD patterns from the cavity, applying both STOPD and PASPD systems, are shown in Figures 2a and $2 \mathrm{~b}$ respectively. It can be observed that the STOPD system finds a number of smaller PDs that are not seen by PASPD because of the larger dynamic range in the STOPD system.

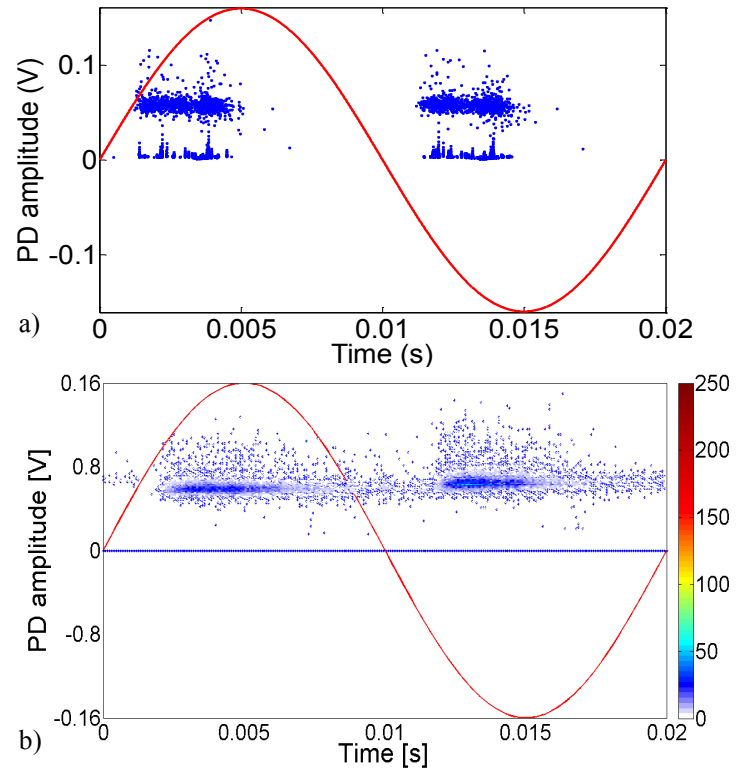

Figure 2. PRPD patterns of cavity PDs at $14 \mathrm{kV}_{\mathrm{pp}}$ sinusoidal voltage detected with the STOPD a) and PASPD b) systems.

Detected PD pulse shape, rise time, equivalent time length and frequency spectrum can generally be used to recognize the time-frequency characteristics of $\mathrm{PD}$ signals. As reported in literature, it is a well-recognized fact that wide band sensors are able to separate different PD sources by pulse shape analysis in time and frequency domain [3436]. This possibility is strongly related to the frequency response of the antenna sensor. Figure 3 shows the time and frequency domain analysis of at PD pulse for both systems. The Fast Fourier transform (FFT) was applied to obtain the Single-Sided Amplitude Spectrum of the PDs. It can be observed that both systems show resonance peaks in the 20 and $30 \mathrm{MHz}$ range and have comparable frequency characteristics. The STOPD system has a bandwidth limited to $50 \mathrm{MHz}$ in these measurements as $100 \mathrm{MHz}$ sample rate is used. Its low frequency content is more suppressed than in the PASPD system, reflecting the resonant detection used. Pulse width in the PASPD system is about $0.15 \mu \mathrm{s}$, whereas the STOPD gives a pulse width of about $0.35 \mu \mathrm{s}$, again due to the resonant detection method.

In the FFT analysis of internal and twisted pair specimens, a prevalent frequency content observed between $10 \mathrm{~Hz}$ and $40 \mathrm{MHz}$. In the motor stator the detected PDs have a shape that oscillates more slowly, as the PD signal path has different characteristics than in the other systems. In this case the measured frequency content is concentrated around $10 \mathrm{MHz}$. These differences in frequency content can provide indications to recognize different PD sources. The PASPD system utilizes FFT and pulse shape cross correlation analysis to separate PDs from noise.

(a)
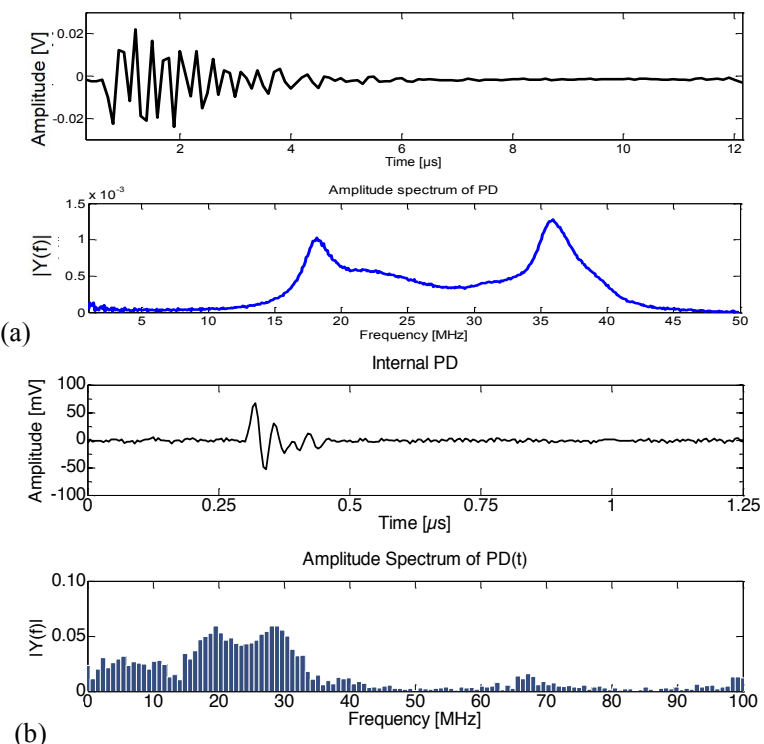

(b)

Figure 3. Cavity PD pulse and its frequency spectrum obtained with 14 $\mathrm{kV}_{\mathrm{pp}}$ (a) sinusoidal voltage with STOP, (b) PASPD( b) systems.

As expected, these results illustrate that both systems were able to detect the PDs from the different sources, each with different properties but obtaining results in good accordance for all the defects.

\subsection{SQUARE VOLTAGE WAVEFORMS}

The two PD acquisition systems presented differ partly in the signal acquisition philosophy, but - equally importantly - also in how the measured data is processed which will be more important for these wave shapes.

The STOPD system is designed specifically to detect PDs at rapidly changing voltage fronts based on a resonant decoupler that suppresses pulses below the lowest resonance frequency, reducing the noises due to remnants and commutations considerably. This procedure together with the use of the stochastic nature of the PD allows to discriminate partial discharge from disturbances with different frequency content as well as phase locked disturbances. All PDs are detected from full-period acquisitions and analyzed for amplitude and timing with high resolution [9]. However at the same time, the resonant decoupler transforms the pulse shape making it difficult to identify possible changes in the PD mechanism without post processing analysis [9].

The PASPD system based on antenna PD sensor, acquire individual PD events utilizing the flat frequency characteristics of the transfer function in the bandwidth between $0 \div 250 \mathrm{MHz}$. However, in this way noises due to remnants and commutations are also acquired without any attenuation. The discrimination process between PD and noises is implemented through the pulse shape, frequency 
content and phase locked analysis. This process employs up to four software filters, in both on and off line mode. Each filter is applied selecting a specific pulse from the pattern with a cursor, then the filter can remove similar pulse shapes applying a cross correlation process with selectable sensitivity [31]. Therefore, the combined advantages of the two PDs measuring systems have allowed a comprehensive examination of the PD phenomenon and to compare output and possible observations. However the use of square wave implies most often the use of semiconductor devices, which may introduce additional noise as will be discussed in the next section.

\subsubsection{Disturbance/noise due to pulses generated by high voltage transistor switch}

The HV switch set-up, illustrated in Figure 1, was used to generate square waveforms. While the polarity shift leaves voltage remnants in the captured data for both PD sensors, the high voltage switch generate some additional high frequency pulses with a fixed repetition rate between each polarity change. In Figure 4, a twisted pair PD pattern with square wave $50 \mathrm{~Hz}$ and rise time $250 \mu$ s shows the presence of some pulses of different origin. In particular, inside the circle, pulses generated by the HTS HV switching at the polarity reversal and inside the square frame pulses generated internally by the HTS switch were identified. These noises are identified by considering that they are constant in phase and are also present at lower voltage than PDIV. Furthermore PD pulse shapes and FFT are different to the noises one. Furthermore, in this case the PDs are larger than the noise, which facilitates the detection considerably. In other cases post-processing methods will be necessary. Thus the problem with additional pulses become more serious at lower frequencies. In particular, for a $50 \mathrm{~Hz}$ square wave, about 32 false PD pulses per period appear, while for a $350 \mathrm{~Hz}$ square wave considerably less. However, these disturbances have a deterministic behavior which make it possible to identify and separate them from the partial discharges by the stochastic PD method using timed gating [8]. Without this signal processing this noise would be present in the measured signal, just as shown in Figure 4 for the antenna system which acquires all pulses simultaneously but processes the data in a completely different way.

The PASPD system detect and acquire all signals together, both disturbances and partial discharges with less distortion of the pulses, due to the flat frequency response of the antenna sensor. The partial discharge analysis must be carried out at the end of the acquisition to identify the actual PDs, employing filters with selectable sensitivity to eliminate undesired signals.

The time and frequency analysis of pulses generated by the HTS HV switching and the time analysis of pulses generated by the DC HV supply are shown in Figure 5 and 6 . The larger pulse signal remnant from the polarity shift contains higher amplitude spectrum in the frequency range between 30 and $40 \mathrm{MHz}$ than the DC supply noise.

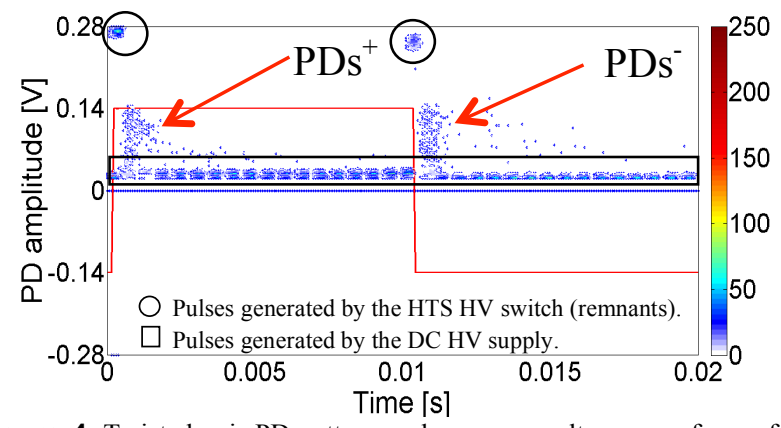

Figure 4. Twisted pair PD pattern under square voltage waveform of 50 $\mathrm{Hz}$ with rise time of $250 \mu \mathrm{s}$. Circle frames and square frames point respectively to pulses generated by the HTS HV switching at polarity reversals and pulses generated internally by the switch.
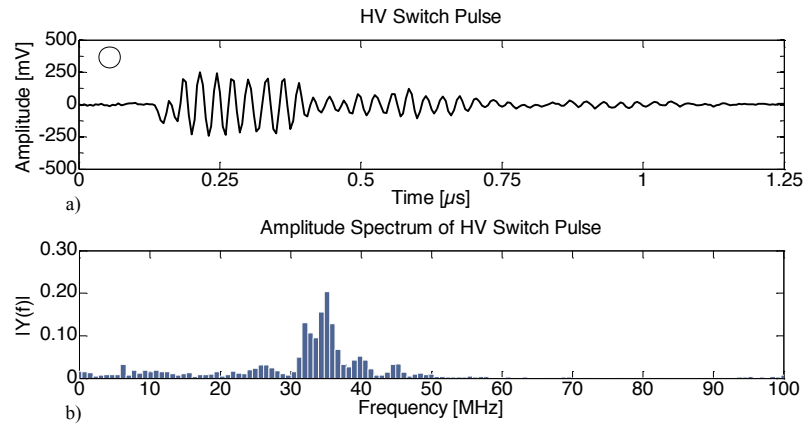

Figure 5. PASPD: identification in time (a) and frequency (b) of pulses generated by HTS HV switching.

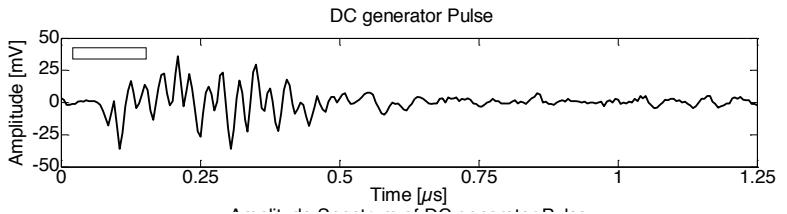

Figure 6. PASPD: Identification in time of pulses generated by HV DC supply.

Otherwise, the PDs shape and repetitive time position of switching noises allow identification between discharges and noises. Separation among different pulse shapes is also possible by applying a cross correlation method.

\subsubsection{Analysis of square waves $50 \mathrm{~Hz} / 350 \mathrm{~Hz}$ at rise times $10 \mu \mathrm{s}$ and $250 \mu \mathrm{s}$}

Sometimes, different PD phenomena can be identified and classified by mean of pattern recognition analysis for sinusoidal waveforms [37]. Using square waveforms, the classical methods for partial discharge identification based on the shape of the discharge pattern is no longer usable. Phase angle $\Delta \varphi$, statistical parameters such as Weibull and others standard statistical parameters are not significant with square waves, so the classical statistical analysis cannot be applied [38].

One of the main characteristics observed for square waves is that most PDs are concentrated at the rising and falling edges of the applied voltage waveform [9]. The adoption of different rise times allows evaluation of its influence of the partial discharge phenomena. In particular, it was observed in all the measurements made, that the phase angle of discharges was lower in the case of square wave with rise time of $10 \mu$ s compared to the case of 250 
$\mu \mathrm{s}$, which is a consequence of PDs occurring only on the edges. As expected the voltage remnant was higher and more oscillatory in the captured data with the shorter rise time than in the case of $250 \mu \mathrm{s}$ rise time. Furthermore, from the tests it was generally observed that the frequency content of the PDs did not change for $50 \mathrm{~Hz}$ or $350 \mathrm{~Hz}$ square waves, as expected.

\section{1) Cavity PD}

The acquisition of cavity discharges with the PASPD system has presented more difficulties than for other defects, especially with the shorter rise time. Internal PDs with a $50 \mathrm{~Hz}$ square wave with $250 \mu$ s rise time have a measured PD amplitude comparable to the switch noise as shown in Figure 7. Therefore, the system acquires both types of pulses. Besides Figure 7 shows the PD pattern with the cavity discharges near the polarity shifts and the switch internal noise spread out over the complete period. It is however still possible to visually distinguish the PD pulses from the noises.

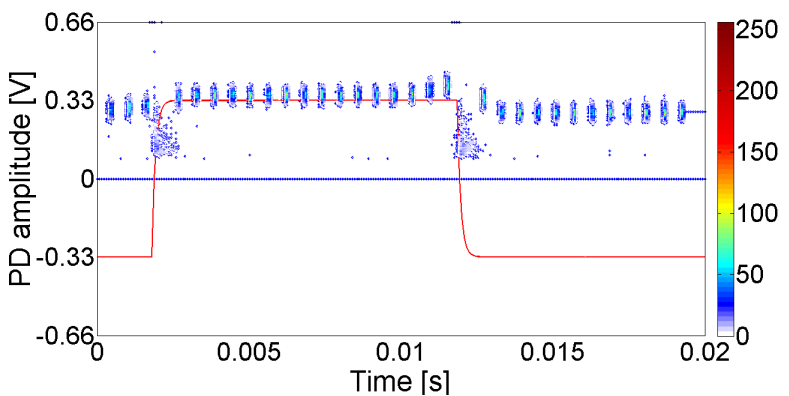

Figure 7. PASPD: Internal PD pattern applying a square wave of $50 \mathrm{~Hz}$ with rise time $250 \mu \mathrm{s}$.

Most PDs are located at the square wave polarity change, but a few are evenly distributed across the period. Here the PD amplitude is comparable or even smaller than the magnitude of the switch noise.

A similar result is obtained at $350 \mathrm{~Hz}$, where only two switch noise pulses appear. In both cases a typical pulse shape is acquired and the amplitude spectrum analysis can be performed. In Figure 8 the PD signal (a) and its amplitude spectrum (b) is shown. The frequency content, although contained mainly between 15 and $40 \mathrm{MHz}$ as in the sinusoidal case, presents a quite different shape distribution (see Figure 3b).
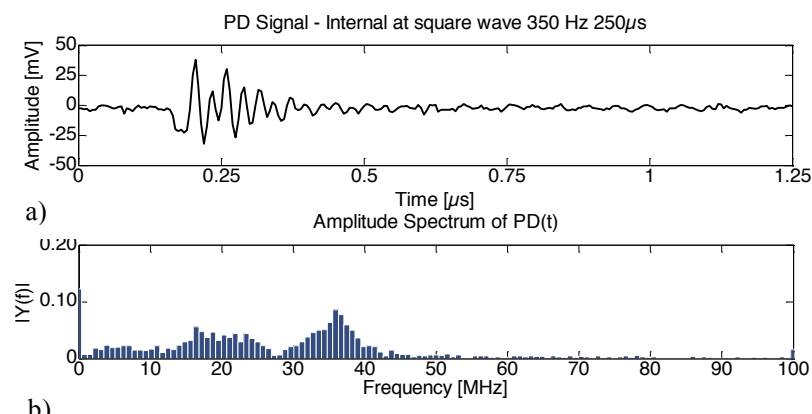

b)

Figure 8. PASPD: Identification in time (a) and frequency (b) Internal PD applying a square wave of $350 \mathrm{~Hz}$ with a rise time of $250 \mu \mathrm{s}$.
An even more demanding condition for the portable PASPD instrument is a measurement when applying a square wave with a rise time of $10 \mu \mathrm{s}$. In such conditions, the instrument was only able to identify the pulses generated by HTS HV switch and with a reduced rise time the PDs appear often superimposed to the voltage remnants as shown in Figure 9. In the Figure it can be observed the $10 \mu$ s voltage rise time (blue line) and the voltage remnant in which PDs are present after the first microsecond (red line). In this case the PDs appears only in the first few microseconds after the remnants while with longer rise times the influence of the voltage remnant is less prominent.

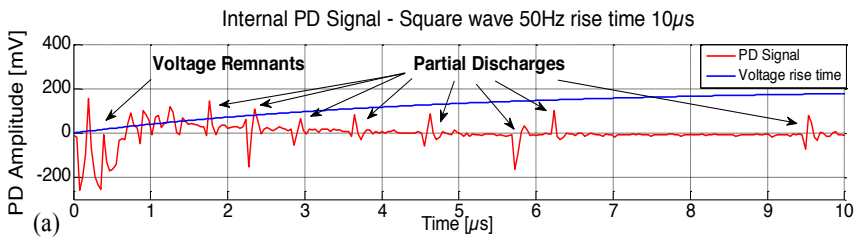

Figure 9. PASPD: Internal partial discharges applying a square wave of $50 \mathrm{~Hz}$ with a rise time $10 \mu$ s superimposed on the voltage remnants.

It is thus observed that the acquisition of PDs become more difficult with the antenna sensor for rise times less than a few tens of microseconds.

With the STOPD system the effect of the voltage's rise time is illustrated in Figure 10, where a comparison among square waves of $10 \mu \mathrm{s}$ and $250 \mu$ s rise times $(50 \mathrm{~Hz})$ and the $50 \mathrm{~Hz}$ sinusoidal waveform are shown. A progressive concentration of PDs to the voltage flank and a high PD magnitude can be observed. In [9] it is however shown that in relation to the rise time, the time delay is increased when the rise time decreases.

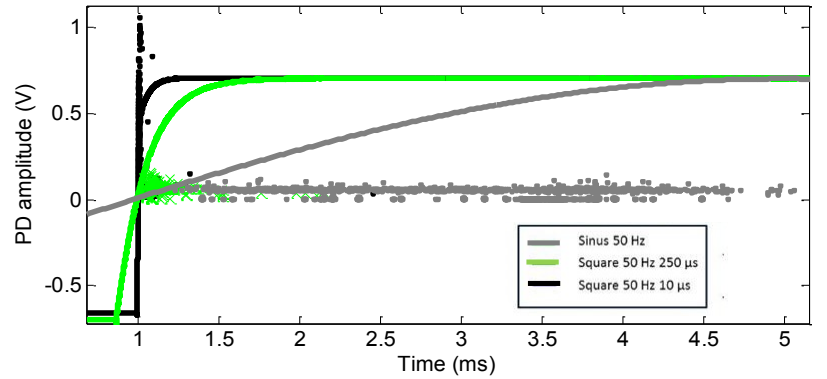

Figure 10. STOPD: Comparison of cavity PD activity during tests with square waves of rise times $10 \mu \mathrm{s}$ and $250 \mu \mathrm{s}(50 \mathrm{~Hz})$ and sinusoidal wave of $50 \mathrm{~Hz}$.

\section{2) Twisted pair PD}

The second defect was represented by surface discharges in twisted pair specimens. With $50 \mathrm{~Hz}$ sinusoidal waveform applied, both systems showed maximum discharge amplitudes up to about $0.2 \mathrm{~V}$ (STOPD) or less. Applying square voltage waveforms at $350 \mathrm{~Hz}$ however do result in higher PD magnitudes at the $10 \mu$ s rise time as illustrated in Figures 11 and 12. Figure 11 illustrates the PD pattern obtained by means of PASPD system when applying 350 $\mathrm{Hz}$ square wave for both 10 and $250 \mu$ s rise times. As can be seen, also in this case there is a strong dependency of the rise time. For the rise time of $10 \mu \mathrm{s}$ (red pattern), PDs are 
predominantly localized on the waveform flanks and their amplitudes are higher. In the second case, rise time of 250 $\mu$ s (blue pattern), the PDs amplitude is lower and conversely the phase angle of discharges increases.

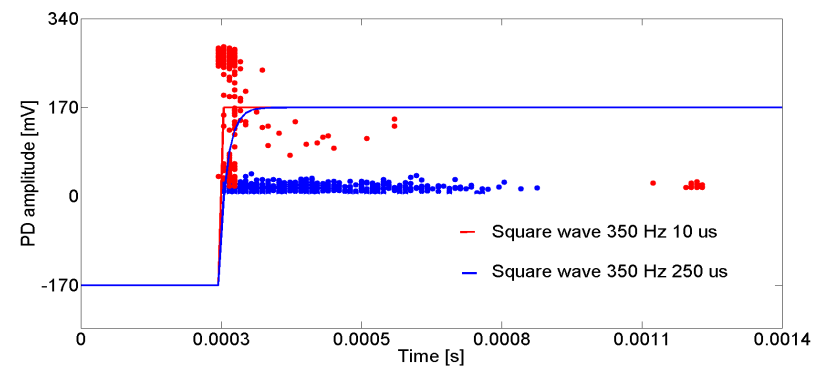

Figure 11. PASPD: Compared twisted pair patterns with square wave 350 $\mathrm{Hz}$ at rise time $10 \mu \mathrm{s}$ and $250 \mu$ s.

Similar results were obtained with STOPD system, see Figure 12. Twisted pair acquisition with square wave 350 $\mathrm{Hz}$ at rise time $10 \mu$ s and $250 \mu$ s respectively have shown the same trend of the patterns. The number of PDs detected follows the number of polarity shifts and the PD amplitudes are larger for the shorter rise time. The longer rise time also reveals more PDs per cycle but with smaller magnitude.

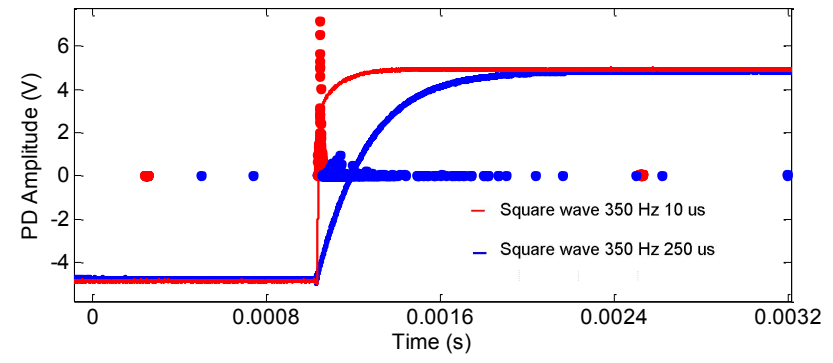

Figure 12. STOPD: Comparison of twisted paid PD activity acquisition with square wave of rise times $10 \mu \mathrm{s}$ and $250 \mu \mathrm{s}(350 \mathrm{~Hz})$.

The PDs that appear in twisted pair test object have overall larger magnitude than the noise, which facilitates the detection process.

\section{3) Motor Stator PD}

The comparisons of PD activity in the motor stator setup, for the $10 \mu \mathrm{s}$ and $250 \mu \mathrm{s}$ waves as well as for the sinusoidal waveform of $50 \mathrm{~Hz}$ are respectively illustrated for PASPD and STOPD systems in Figures 13 and 14. Here again a progressive increase in PD amplitudes can be observed for shorter rise times, whereas their magnitudes for the longer rise time and the sinusoidal waveform are about the same. The dynamic range used in the STOPD system is however larger. The PD magnitudes close to the polarity shift are here larger than the switching noise, which better facilitates the data analyses that are done on-line. For PASPD system the triggering level is set below the switching noise and thus more challenging to resolve the PDs, which requires an additional post processing to quantify the actual exposure.

The multitude of PDs appearing in the captured trace for this test object makes it impossible to resolve all PDs [9], thus only the ones detected with the STOPD system are indicated.

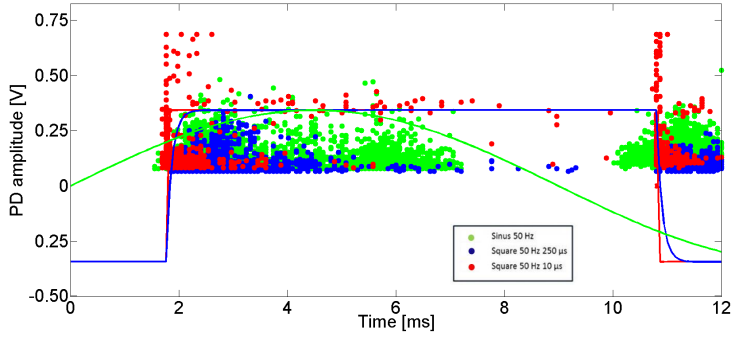

Figure 13. Comparison of motor stator PD activity during PASPD tests with square waves of rise times $10 \mu \mathrm{s}$ and $250 \mu \mathrm{s}(50 \mathrm{~Hz})$ and sinusoidal wave of $50 \mathrm{~Hz}$. Note that, in these tests, the sinusoidal peak amplitude of the applied voltage is somewhat higher than the square waves.

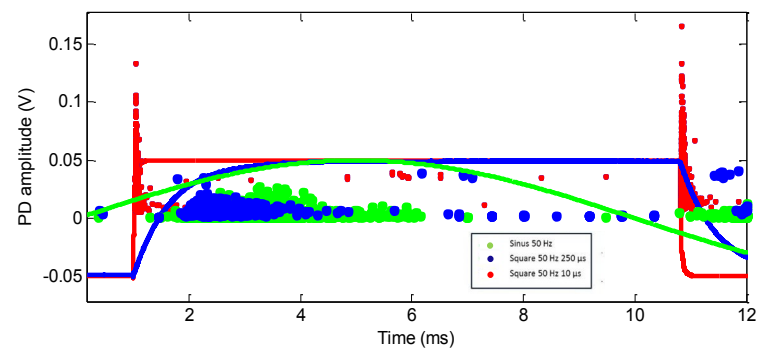

Figure 14. Comparison of motor stator PD activity during STOPD tests with square waves of rise times $10 \mu \mathrm{s}$ and $250 \mu \mathrm{s}(50 \mathrm{~Hz})$ and sinusoidal wave of $50 \mathrm{~Hz}$.

Figure 15 exemplifies a stator motor PD pulse shape recorded by PASPD and STOPD systems.

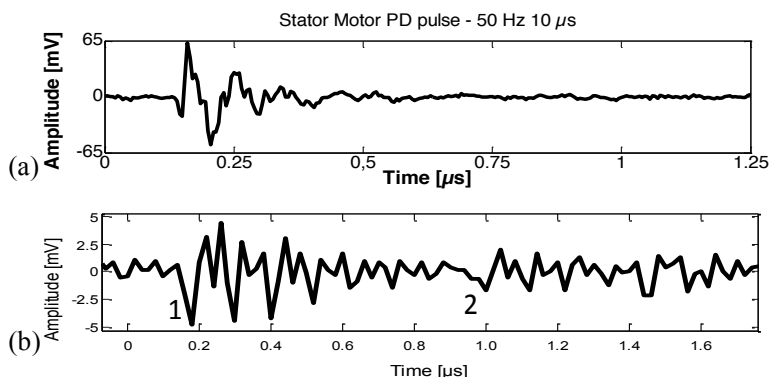

Figure 15. Motor stator PD pulses at $50 \mathrm{~Hz}$ square wave of $10 \mu$ s rise time; (a) obtained with PASPD system, (b) obtained with STOPD system with 2 PD events indicated. Note that the same PD event is not used in both figures.

\section{Discussion}

It has been shown by means of the presented study that both the tested PD measurement systems, PASPD and STOPD, were able to measure the maximum amplitudes of PDs occurring above the switching noise. The increase in PD magnitudes for the shorter rise time was also recorded by means of both systems for the surface type of discharges (twisted pair and motor stator) while some problems appeared with detecting cavity PDs by PASPD system. In contrast, STOPD system could record the cavity PDs in the same way as for the sinusoidal exposure.

The presented tests were performed with the aim to observe how different rise times of voltage waveforms influence PD characteristics of various types of objects, as observed in [9, 19], that the use of steeper waveforms results in larger PD magnitudes and appearing at higher 
(applied) voltage levels. Several other indications for possible changes in PD mechanisms has been observed, such as shorter rise time of the PD itself for the steeper waveforms. We thus below discuss how a measurement system should be designed to obtain relevant information under challenging conditions.

The standard sinusoidal $50 \mathrm{~Hz}$ waveform tests are presented for comparison to illustrate the PD behavior of the used sources and to show that PASPD and STOPD systems may well be used, albeit with different parameters in the analyses. This is because the STOPD system, designed for power electronic waveforms, provides additional analysis options, and only a few of them have been here presented. For the PASPD general-purpose system, the analysis parameters are of standard type, obtainable by means of similar commercial systems.

An important feature in the present context is the pulse resolution time, which must be considerably smaller than the voltage rise time to facilitate detection of multiple PDs at rising voltage flanks. To handle synthesized waveforms such as PWM, additional features are required, such as several time scales to keep track of PD positions in relation to both the switching frequency and the generated lowfrequency sinusoidal waveform; such features are not discussed here, see [9] for a more complete description.

There are many design choices to make when a PD system is built. Unfortunately, only the sensing method is mainly given attention while other important design features that affect the final result are overlooked. A simple example of the influence of design choices is that the maximal frequency used, $350 \mathrm{~Hz}$, was dictated by the general purpose system (PASPD) as it had difficulties to synchronize to frequencies higher than about $500 \mathrm{~Hz}$. This limitation comes solely from the design and some additional adjustments may enable its synchronization to higher frequencies.

Basic to any method for detecting PDs when applying square shaped voltages is the ability to suppress the highfrequency components of the test voltage, so that these are not mistaken for PDs or inhibit a sensitive detection. Several methods are needed here, nevertheless short voltage rise times will impose problems at least when the rise time is so short that it is comparable to the PD duration, which often is a few ns. The future use of $\mathrm{SiC}$ technology may be an example of such challenging conditions and there future work is required. The primary method is to suppress low frequencies by the analogue sensing circuit which is done in both systems by different means. The resonant coupler used by STOPD system suppress $1 \mathrm{MHz}$ four orders of magnitude below the first resonance around $10 \mathrm{MHz}$ [9]. The antenna sensor is equipped with a high pass filter having a cut-off frequency of $500 \mathrm{kHz}$. Shorter rise times demand higher cut-off frequencies. High cut-off frequencies pose however a risk of not being able to detect PDs in all situations as PD sources with $\mu$ s duration has been observed [39], therefore other methods are required to handle those cases.
To improve pulse resolution, particularly at short rise times (less than $10 \mu \mathrm{s}$ ), it is important that the duration of PD pulses are as short as possible. Here, the resonant properties of STOPD system provide longer pulse duration than the wide-band acquisition in PASPD system. A larger band-width generally implies lower sensitivity, however. This point certainly represents a goal for an optimized measurement system.

The STOPD system is designed to suppress the remnants of the voltage signal after the analogue sensor by utilizing the fact that these remnants are repeatable while PDs are not. This is a very effective method, but requires that both PD signals and voltage remains are resolved in digitization. High-resolution digitizers are thus an advantage with this technique as the PD signal can then be allowed to be hundred times smaller than the voltage remnants and still be detected. The general-purpose system does not employ stochastic analysis even though it may be favorable for AC waves in some conditions [2].

Another method to suppress disturbances is to use gating, i.e., PD detection is turned off under certain conditions. Such functionality is available in many commercial PD detection systems for AC, where an extra channel is used to pick up disturbances. In the present case, the disturbances are generated by the voltage source and could ideally be suppressed by the stochastic technique. This requires however that the disturbing signals are adequately digitized and stable in time. Neither condition applies in STOPD system: the sampling rate is too low to resolve the switching noise in enough detail and there is further an inherent time jitter in the switch of the order of a $1 \mu \mathrm{s}$. Thus the system uses timed gating to suppress these disturbances. The gating times are determined from a measurement below PD inception, prior to the actual measurement. Such a timed gating facility is probably required for any PD system that should be used for power electronic generated waveforms.

An important design difference between the two systems studied is how a PD signal is acquired. Many digital PD systems, including the presently studied PASPD system, trigger acquisition when the analogue signal is above a preset limit that defines the smallest PD detectable. When triggered, a PD signal is recorded for a specific time, in this case generally $1.25 \mu \mathrm{s}$, and the PD parameters are extracted from this recording. Besides the trigger approach however, PASPD system can as an additional feature suppress unwanted signals by applying filters, based on a cross correlation process with selectable sensitivity, removing all pulses with similar shape. In contrast, the stochastic analysis requires much longer recordings, which are triggered on the applied voltage to properly record the voltage remains. PDs are detected by software in these records after subtraction of the repetitive signal. In addition to enable the stochastic treatment this gives also greater flexibility in the PD parameter analysis and makes timed gating possible. The PD trigger level can be determined from a signal noise estimate and the duration can be variable. With careful design of the PD analysis algorithm, 
it is possible to detect the next PD signal before the previous has fully decayed.

Post-acquisition detection of PDs in a longer recording is thus a requisite for a $\mathrm{PD}$ system used with rapidly changing voltages as this gives the shortest possible time between detected PD events. As seen in Figure 9 (10 $\mu$ s rise time \& $10 \mu \mathrm{s}$ recording in antenna system), PASPD system can record numerous PDs occurring on the voltage flank but has presently limited capability to analyze them individually.

\section{Conclusions}

It is demonstrated that there are a number of features, important for effective detection of PD signals appearing at rapidly changing test voltages from power electronic devices. The main requirement is the ability to synchronize to substantially higher frequencies than the normal power frequency. A more complete analysis of full periods, such as appearing at PWM shapes requires the use of several time scales. Another basic requirement is the ability to effectively supress the high frequency content in the test voltage. Analogue or digital frequency domain filters play can be utilized, but cannot be expected to handle this task fully satisfactorily by themselves at very short rise times. Therefore, to enhance PD detectability, other techniques are required, such as the time domain stochastic filtering that was employed here.

Another observation is that it is preferable to detect PDs in digitally recorded traces instead from analogue signals, as this enables the highest possible PD time resolution and eliminates the hardware dead-time.

This work has also demonstrated that PDs can be detected by various sensing methods with comparable sensitivity. It is thus more the design features mentioned above that may enable a PD system to be used for power electronic waveforms than the sensing principle as in all insulation systems tested it was shown possible to detect the presence of PDs and observe changes in amplitude for square shaped voltages.

\section{ACKNOWLEDGMENT}

Chalmers' Area of Advance in Energy is acknowledged for financially supporting this research project.

\section{REFERENCES}

[1] C. Nyamupangedengu, I. R. Jandrell, "Partial discharge spectral response to variations in the supply voltage frequency," IEEE Trans. Dielectr. Electr. Insul., vol. 19, pp. 521-532, April 2012.

[2] T. Bengtsson, E. Lindell, T. Hammarstrom, "Stochastic detection of partial discharges," IEEE Trans. Dielectr. Electr. Insul., vol. 20, pp. 2203-2211, Dec. 2013.

[3] A. Rodrigo Mor, P. H. F. Morshuis, J. J. Smit. "Comparison of charge estimation methods in partial discharge cable measurements," IEEE Trans. Dielectr. Electr. Insul., vol. 22, pp. 657-664, April 2015.

[4] D. Fabiani, G. C. Montanari, "The effect of voltage distortion on ageing acceleration of insulation systems under partial discharge activity," IEEE Electr. Insul. Mag., vol. 17, pp. 24-33, May 2001.

[5] S. Chandrasekar, G. C. Montanari, "Analysis of partial discharge characteristics of natural esters as dielectric fluid for electric power apparatus applications," IEEE Trans. Dielectr. Electr. Insul., vol. 21, pp. 1251-1259, June 2014
[6] B. Florkowska, J. Roehrich, P. Zydron, M. Florkowski, "Measurement and analysis of surface partial discharges at semisquare voltage waveforms," IEEE Trans. Dielectr. Electr. Insul., vol. 18, pp. 990-996, Aug. 2011.

[7] A. Madonia, P. Romano, T. Hammarström, S. M. Gubanski, F. Viola, A. Imburgia, "PD characteristics at Square Shaped Voltages Applying Two Different Detecting Techniques," in Proceedings of IEEE Conference on Electrical Insulation and Dielectric Phenomena (CEIDP), 2016, pp. 247-250.

[8] E. Lindell, "Partial Discharges at Repetitive Rapidly Changing Voltages," PhD Dissertation, Chalmers University of Technology, Göteborg, Sweden, 2009.

[9] T. Hammarström, "Partial Discharges at Fast Rising Voltages," PhD Dissertation, Chalmers University of Technology, Göteborg, Sweden, 2014.

[10] E. Gulski, "Digital analysis of partial discharges," IEEE Trans. Dielectr. Electr. Insul., vol. 2, pp. 822-837, Oct. 1995.

[11] F. Guastavino, A. Dardano, "Life tests on twisted pairs in presence of partial discharges: Influence of the voltage waveform," IEEE Trans. Dielectr. Electri. Insul., vol. 19, pp. 45-52, Feb. 2012.

[12] B. Florkowska, M. Florkowski, J. Roehrich, P. Zydron, "The influence of PWM stresses on degradation processes in electrical insulation systems," in Proceedings of IEEE Conference on Electrical Insulation and Dielectric Phenomena (CEIDP), 2010, pp. $1-4$.

[13] A. Cavallini, D. Fabiani, G. C. Montanari, "Power electronics and electrical insulation systems Part 1: Phenomenology overview," IEEE Electrical Insulation Magazine, vol. 26, pp. 7-15, Jun. 2010.

[14] F. P. Espino-Cortes, E. A. Cherney, S. H. Jayaram, "Impact of inverter drives employing fast-switching devices on form-wound AC machine stator coil stress grading," IEEE Electrical Insulation Magazine, 23, pp.16-28, Jan. 2010.

[15] P. Wang, A. Cavallini, G. C. Montanari, G. Wu, "Effect of rise time on PD pulse features under repetitive square wave voltages," IEEE Trans. Dielectr. Electri. Insul., vol. 20, pp 245-254, Feb. 2013.

[16] P. Wang, A. Cavallini, G. C. Montanari, "The effects of square wave voltage rise time on PD statistics in time and frequency domain," in Proceedings of IEEE Conference on Electrical Insulation Conference (EIC), 2015, pp. 262-265.

[17] P. Wang, A. Cavallini, G. C. Montanari, "The influence of square voltage rise time on partial discharge spectra," in Proceedings of IEEE Electrical Insulation and Dielectric Phenomena (CEIDP), 2012, pp. 129-132.

[18] E. Lindell, T. Bengtsson, J. Blennow, S. M. Gubanski, 'Influence of rise time on partial discharge extinction voltage at semi-square voltage waveforms," IEEE Trans. Dielectr. Electri. Insul., Vol. 17, pp 141-148, Feb. 2010.

[19] T. Hammarström, T. Bengtsson, J. Blennow, S. M. Gubanski, "Evidence for changing PD properties at short voltage rise times," IEEE Trans. Dielectr. Electri. Insul., Vol. 18, pp. 1686-1692, Oct. 2011.

[20] P. Romano, F. Viola, R. Miceli, B. D’Agostino, C.Spataro, "Partial discharges on IGBT modules: Are sinusoidal waveforms sufficient to evaluate behaviour?," in Proceedings of IEEE Conference on Electrical Insulation and Dielectric Phenomena (CEIDP), 2014, pp. 224-227.

[21] G. C. Montanari, F. Negri and F. Ciani, "Partial Discharge and life behavior of rotating machine wire insulation under PWM waveforms: The influence of inverter characteristics," in Proceedings of IEEE Electrical Insulation Conference (EIC), 2017, pp. 161-164.

[22] G. Skibinski, W. Maslowski and J. Pankau, "Installation considerations for IGBT AC drives," in Proceedings of IEEE Annual Textile, Fiber and Film Industry Technical Conference, 1997, pp. 6. 12.

[23] D. Xiang, L. Ran, P. Tavner, S. Yang, A. Bryant and P. Mawby, "Condition Monitoring Power Module Solder Fatigue Using Inverter Harmonic Identification," in IEEE Trans. on Power Electronics, vol. 27, pp. 235-247, Jan. 2012.

[24] Y. Shibuya, S. Matsumoto, T. Konno, K. Umezu "Electromagnetic waves from partial discharges in windings and their detection by patch antenna," IEEE Trans. Dielectr. Electri. Insul., Vol. 18, pp. 2013-2023, Dec. 2011.

[25] G. Robles, M. Sánchez-Fernández, R. Albarracin Sanchez, Rojas- M. V. Moreno, E. Rajo-Iglesias, J. M. Martínez-Tarifa, "Antenna 
parametrization for the detection of partial discharges," IEEE Trans. Instrument. Measure., vol. 62, pp. 932-941, May 2013.

[26] R. Candela, A. Di Stefano, G. Fiscelli, G. C. Giaconia, "Portable Partial Discharge Detection Device", Patent EP2297589/ WO2009150627, June 11, 2009.

[27] R. Candela, A. Contin, "A portable instrument for the location and identification of defects generating PD," in Proceedings of IEEE Electrical Insulation Conference (EIC), 2011, pp. 469-473.

[28] Ala G., Candela R., Romano P., Viola, F. "Simplified hybrid PD model in voids," in Proceedings of IEEE International Symposium of Diagnostics or Electric Machines, Power Electronics \& Drives (SDEMPED), 2011, pp. 451-455.

[29] M. L. Di Silvestre, R. Miceli, P. Romano, F. Viola, "Simplified hybrid PD model in voids: Pattern validation," in Proceedings of IEEE Fourth International Conference on Power Engineering, Energy and Electrical Drives (POWERENG), 2013, pp. 1807-1813.

[30] F. Viola, P. Romano, "Building Partial Discharge Signal Wireless Probes," in Progress in Compact Antennas, L. Huitema, InTech, 2014, pp.177-203.

[31] G. Ala, R. Candela, F. Viola, "Detection of Radiated EM Transients by Exploiting Compact Spherical Antenna Features", Recent Patents on Electrical \& Electronic Engineering, vol. 4, pp. 202-208, Sep. 2011.

[32] F. Viola, P. Romano, R. Miceli, A. Contin, "Performance of the shape of Partial Discharge signal wireless probes", in Proceedings of IEEE Fourth International Conference on Power Engineering, Energy and Electrical Drives (POWERENG), 2013, pp. 1801-1806.

[33] R. Candela, A. Di Stefano, G. Fiscelli, S. F. Bononi, L. De Rai, "A novel partial discharge detection system based on wireless technology," in Proceedings of AEIT Annual Conference, 2013, pp. $1-6$.

[34] A. Cavallini, M. Conti, A. Contin, G. C. Montanari, "Advanced PD Inference in On-Field Measurements. Part 2: Identification of Defects in Solid Insulation Systems," IEEE Trans. Dielectr. Electri. Insul., vol. 10, pp. 528-538, June 2003.

[35] G.Robles, J.M. Martínez-Tarifa, M.V. Rojas-Moreno, R. Albarracín, J. Ardila-Rey, "Antenna selection and frequency response study for UHF detection of partial discharges," in Proceedings of IEEE International Instrumentation and Measurement Technology Conference (I2MTC), 2012, pp. 1496-1499.

[36] A. Haddad and D. Warne, "Advances in High Voltage Engineering". London: IET Press, 2004, pp. 668

[37] A. Cavallini, G. C. Montanari, F. Puletti and A. Contin, "A new methodology for the identification of PD in electrical apparatus: properties and applications, “ in IEEE Trans. Dielectr. Electri. Insul., vol. 12, pp. 203-215, April 2005.

[38] R. Candela, G. Mirelli, R. Schifani "PD Recognition by Means of Statistical and Fractal Parameters and Neural Network," IEEE Trans. Dielectr. Electri. Insul., vol. 7, pp. 87-94, Feb. 2000.

[39] H. Debruyne and A. Lesaint, "About the significance of PD measurements in liquids," IEEE Trans. Dielectr. Electri. Insul., Vol. 10, pp. 385-392, June 2003.

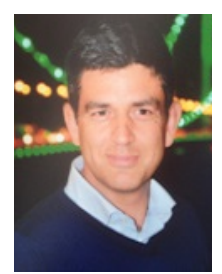

Pietro Romano (M'14-SM'17) received the M.Sc. and Ph.D. degrees in Electrical Engineering from University of Palermo, Italy, in 1993 and 1998, respectively. From 1998 to 2001, he worked at CRES Centre of Electronic Research on Sicily as researcher on partial discharge measurements. From 2001 he is a researcher at the Department of Electrical Engineering at the University of Palermo and teaches Basic Electrical Engineering, Electrotechnics and insulating materials. His research activity is mainly in the field of insulating systems diagnosis, partial discharge measurements, HV Systems, multifactor stress effects and electric field simulations, partial shaded PV Systems. He is the head of the L.E.PR.E. HV laboratory of Palermo University.

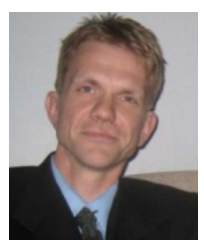

Thomas J.Å. Hammarström received the M.Sc. degree in engineering physics from Chalmers University of Technology in 1998. Between 98 and 09 he worked as a research engineer with developing new high energy ignition and control system for Opcon $\mathrm{AB}$ and Phelon Euro $\mathrm{AB}$ respectively. He received the Ph.D. degree in High Voltage Engineering at Chalmers University of Technology in Gothenburg, Sweden in 2014, where his main focus was on analysing partial discharges. He is currently working as researcher and lecturer at the Department of Materials and Manufacturing Technology, Chalmers University of Technology.

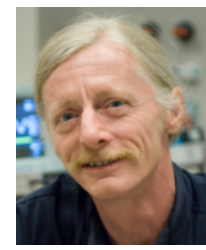

Tord Bengtsson received the $\mathrm{Ph} . \mathrm{D}$. degree in mathematical physics from Lund Institute of Technology in 1984 and continued working with theoretical nuclear physics until 1991, when he joined ABB Corporate Research in Västerås, Sweden. Since then, he has been actively engaged in developing diagnostic methods for high voltage apparatuses and systems. Some particular interests are acoustic methods, partial discharges and signal processing. In 2006, he was appointed as a part time professor in High Voltage Engineering at Chalmers University of Technology in recognition of his efforts to develop research on insulation properties under voltages with fast rise times.

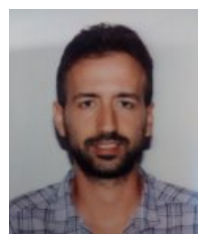

Antonino Imburgia was born in Palermo, Italy, on 28 April 1987. He received the M.Sc. degree in electrical engineering from the University of Palermo in 2014. From 2015 he is a Ph.D. student at the Department of energy, information engineering and mathematical models (DEIM) at the University of Palermo. His current research is in the field of HVDC, distribution of space charge in solid dielectrics and its behavior, dielectrics and electrical insulating systems diagnosis, space charge and partial discharge measurements.

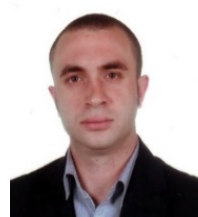

Antonino Madonia was born in Palermo, Italy on 7 July 1985. He received the MSc. degree in Electrical Engineering from University of Palermo, Palermo, Italy, in 2014. In 2015 he joined the Department of Energy, Engineering and Mathematical Models (DEIM) of the University of Palermo as PhD Student. His research interests are in the field high voltage systems, partial discharges and renewable energy.

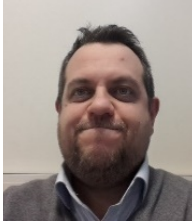

Fabio Viola received the MSc. degree in Electrical Engineering from University of Palermo, Palermo, Italy, in 2002 and the Ph.D. in Electrical Engineering from the same institute in 2006.

In 2008 Dr. Viola joined the Department of Electrical, Electronic and Telecommunication Engineering of the University of Palermo as researcher. His research interests are in the field of electromagnetic compatibility, high voltage, partial discharges, renewable energy and energy harvesting.

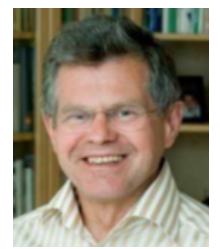

Stanislaw M. Gubanski (M'89-SM'90-F'01) received the M.Sc. (high voltage engineering) and Ph.D. degrees (material science) from the Technical University of Wroclaw, Poland, in 1973 and 1976, respectively. He was a Research Fellow at the University College of North Wales Bangor, U.K from 1976 to 1977 , and a senior lecturer at the Technical University of Wroclaw, Wroclaw, Poland, from 1977 to 1988. Afterwards he was associate professor (1989-1996) at the Royal Institute of Technology, Stockholm, Sweden. Currently, he is professor in High Voltage Engineering at the Department of Materials and Manufacturing Technology, Chalmers University of Technology. He is a Senior Associate Editor of the IEEE Transactions on Dielectrics and Electrical Insulation. 